\title{
Effect of ankle-foot orthosis on roll-over shape in adults with hemiplegia
}

\author{
Stefania Fatone, PhD, BPO(Hons); ${ }^{*}$ Andrew H. Hansen, PhD \\ Northwestern University Prosthetics Research Laboratory and Rehabilitation Engineering Research Program, \\ Department of Physical Medicine and Rehabilitation, Feinberg School of Medicine, Northwestern University, \\ Chicago, IL; Jesse Brown Department of Veterans Affairs Medical Center, Chicago, IL
}

\begin{abstract}
Ankle-foot orthoses (AFOs) are intended to improve toe clearance during swing and ankle position at initial contact (IC) and midstance. Changes that lead to improved ankle-foot kinematics may result in a more biomimetic rollover shape (ROS). ROS is the effective geometry to which the ankle-foot complex conforms between IC and contralateral IC. An effective ROS during gait may facilitate forward progression. This study investigated the effect of an AFO on ROS in adults with hemiplegia following stroke. Kinematic and force data were recorded from 13 people with hemiplegia and 12 controls. Hemiplegic subjects walked at a self-selected speed with and without an articulated AFO with plantar flexion stop. For the involved limb, the AFO significantly increased the ROS arc length (from $32.6 \%$ to $55.7 \%$ of foot length [FL]) and arc radius ( $67.4 \%$ to $139.3 \%$ of FL) and significantly altered the sagittal plane location of the first center of pressure (COP) point, moving it posterior to the ankle center $(-1.2 \%$ to $-20 \%$ of FL) ( $p<0.002$ for all comparisons). However, when hemiplegic patients walked with an AFO, their mean arc radius was greater, mean arc length less, and the first COP point further posterior than those of control subjects.
\end{abstract}

Key words: AFO, ankle-foot orthosis, gait analysis, hemiplegia, orthoses, orthotic device, rehabilitation, rocker, roll-over shape, stroke.

\section{INTRODUCTION}

Hemiplegic gait is characterized by slow, labored, and uncoordinated limb movements [1]. Variability in area of lesion, degree of pathology, and period of recovery contributes to differences in gait among people with hemi- plegia. Residual muscle weakness, abnormal movement synergies, and spasticity result in altered gait patterns and contribute to poor balance, greater risk of falling, and increased energy expenditure during walking [2]. People with hemiplegia have poor single-limb balance and difficulty controlling forward progression [3]. Their gait is asymmetrical with significantly reduced involved lowerlimb range of motion (ROM) and walking speed. Gait changes from those of nondisabled persons walking at self-selected speeds result not only from the inability to selectively control movement but also from the slow speed of movement. Limited hip, knee, and ankle motion resulting in a stiff-legged gait is frequently reported. An equinovarus deformity is often present and compromises heel strike when the person is walking [4].

Perry described normal function of the foot and ankle as the combination of three sequential rockers: the heel,

\footnotetext{
Abbreviations: $3-\mathrm{D}=$ three-dimensional, $\mathrm{AFO}=$ ankle-foot orthosis, $\mathrm{COP}=$ center of pressure, $\mathrm{DF}=$ dorsiflexion, $\mathrm{FL}=$ foot length, $\mathrm{GRF}=$ ground reaction force, $\mathrm{IC}=$ initial contact, $\mathrm{PF}=$ plantar flexion, R\&D = Research and Development, $\mathrm{ROM}=$ range of motion, ROS = roll-over shape, $\mathrm{VA}=$ Department of Veterans Affairs, VACMARL = VA Chicago Motion Analysis Laboratory.

*Address all correspondence to Stefania Fatone, PhD, BPO(Hons); Northwestern University Prosthetics Research Laboratory and Rehabilitation Engineering Research Program, 345 E Superior Street, RIC 1441, Chicago, IL 60611; 312-238-6538; fax: 312-238-6510.

Email: s-fatone@northwestern.edu

DOI: 10.1682/JRRD.2006.08.0090
} 
ankle, and forefoot [5]. She suggested that during the stance phase of nondisabled subjects, progression over the supporting foot is assisted by these three rockers. Roll-over shape (ROS) is defined as the effective geometry to which the ankle-foot complex conforms between initial contact and opposite initial contact [6] and represents the integrated effect of the ankle-foot rockers described by Perry [5] that occurs during the same period. Research has shown that the ankle-foot complex of nondisabled persons creates an effective ROS during normal gait that is essentially invariant to added weight to the torso, walking speed, and footwear [6-8].

When pathologies such as hemiplegia are present, ankle-foot function is disrupted and an ankle-foot orthosis (AFO) may be worn in an attempt to restore function. AFOs have been reported to improve toe clearance during swing and ankle position at initial contact (IC) [4,9-10]. Other reports have suggested possible influences of an AFO on the knee [11-16]. Improved anklefoot kinematics may also result in a more biomimetic ROS. Obtaining a particular ROS seems desirable as a goal since the nondisabled ankle-foot complex adapts to various conditions, such as walking speed, to maintain a consistent ROS radius and orientation. For hemiplegic subjects, use of an AFO may increase center of pressure (COP) excursion and also improve the ROS, i.e., making ROS more closely resemble that of nondisabled subjects. An effective ROS during gait may facilitate forward progression [17]. This study investigated the effect of an AFO on ROS in adults with hemiplegia following stroke.

\section{METHODS}

We initially assessed all participants with hemiplegia by documenting passive ankle ROM using a goniometer while the subject lay supine with the knee flexed and then extended. Subjects were then cast by a qualified orthotist for a custom, thermoplastic, articulated AFO with $90^{\circ}$ plantar flexion stop, free dorsiflexion, and full-length foot plate (Figure 1). All AFOs were fabricated with $3 / 16$ in. polypropylene and Tamarack Flexure Joints (Becker Orthopedic, Troy, Michigan). A dorsal ankle strap was attached to the AFO when needed on the basis of clinical judgment. The impression was taken with the ankle in a neutral position; i.e., with the tibia and foot aligned at a $90^{\circ}$ angle. Footwear was standardized for subjects with hemiplegia, with each participant receiving a pair of

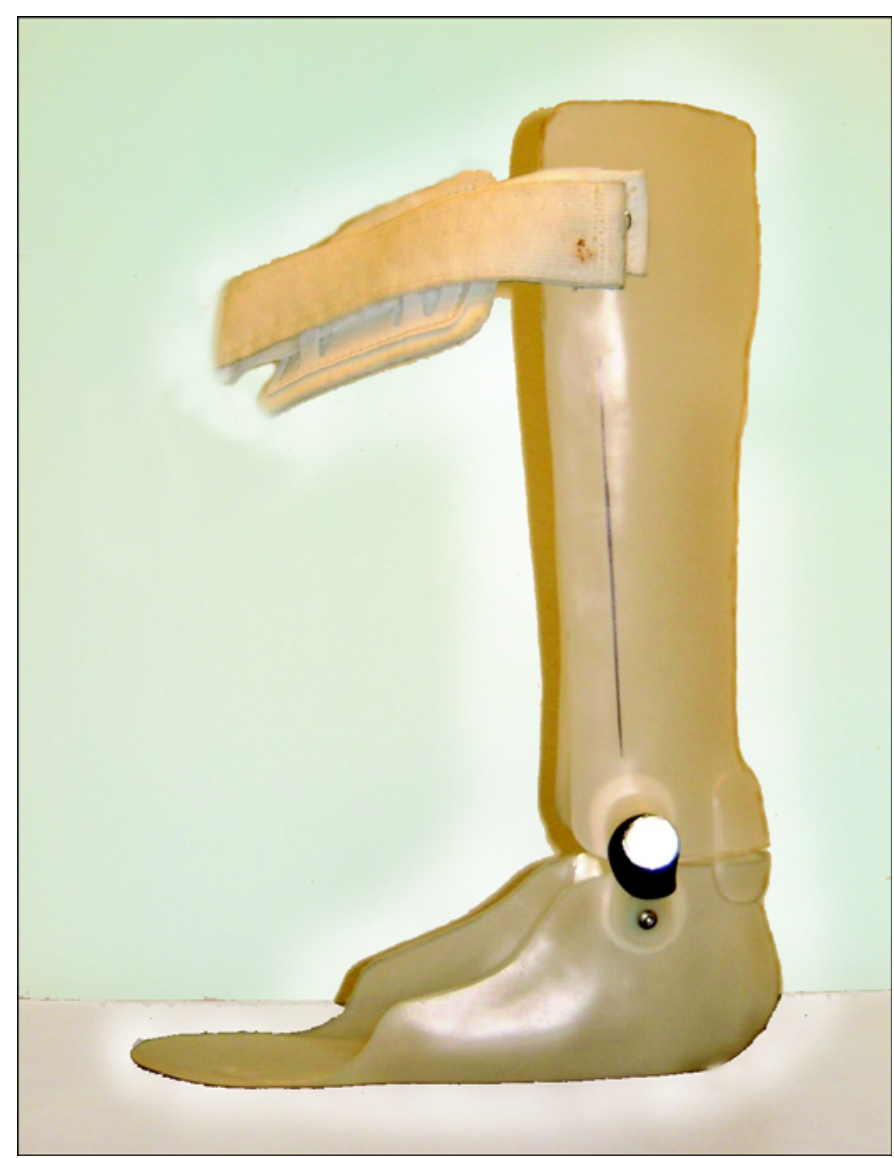

\section{Figure 1.}

Custom, thermoplastic, articulated ankle-foot orthosis (AFO) with $90^{\circ}$ plantar flexion stop, free dorsiflexion, and full-length foot plate received by all subjects. Ankle marker shown attached to top screw of ankle joint. Dorsal ankle strap (not shown) was attached to AFO when needed on basis of clinical judgment.

extra-depth leather shoes (PW Minor \& Son, Inc, Batavia, New York) with a $1.1 \mathrm{~cm}$ heel-to-forefoot sole thickness difference. The shoes had the effect of anteriorly tilting the tibia in the AFO approximately $5^{\circ}$ from the vertical when the plantar flexion stop was maximally engaged. Subjects were allowed 2 weeks of accommodation to the orthosis prior to data collection.

We acquired all gait data at the Department of Veterans Affairs (VA) Chicago Motion Analysis Research Laboratory (VACMARL), which is equipped with an eightcamera real-time motion capture system (Motion Analysis Corporation, Santa Rosa, California) recording kinematic data at $120 \mathrm{~Hz}$ and six force-plates (Advanced Mechanical Technology, Inc, Watertown, Massachusetts) embedded flush in the floor of a $10 \mathrm{~m}$ walkway and recording ground 
reaction forces (GRFs) at $960 \mathrm{~Hz}$. We then resampled force data at $120 \mathrm{~Hz}$ (by taking every eighth data point) to synchronize with the motion data. During the gait analyses, reflective markers were taped to the skin over palpable anatomic landmarks. We used the Helen Hayes marker set, which allowed us to acquire three-dimensional (3-D) data from the pelvis and both lower limbs. Retroreflective surface markers were located on the shoe over the dorsum of the foot at the level of the third metatarsal phalangeal joint, on the shoe over the posterior calcaneus, lateral malleoli, lateral epicondyles of the knees; on right and left anterior superior iliac spines; and on the sacrum midway between the posterior superior iliac spines. Wand markers were placed on the lateral aspects of the thigh and calf. When used, the AFO obscured the landmarks required for estimation of the physiologic ankle joint axis. Because of this constraint, markers were screwed into the mechanical anklejoint of the AFO (Figure 1). We placed the calcaneal marker $1 \mathrm{~cm}$ higher than the toe marker with respect to the ground to account for the difference in the heel and forefoot sole thickness of the shoe. For consistency, the same laboratory personnel placed all markers on subjects on all occasions.

The Northwestern University Institutional Review Board approved this study and informed consent was obtained from each individual prior to their participation. Subjects, using the same shoes, walked at their normal self-selected speed with and without the custom AFO. Trials were collected until three clean force plate strikes (defined as a single foot in contact with the force plate and entirely within the force-plate boundaries) were recorded for each foot. We used EVa RealTime software (Motion Analysis Corporation) to determine the 3-D position of each marker relative to the laboratory coordinate system during each frame of each trial. The raw coordinate data were filtered with a Butterworth second-order bidirectional low-pass filter with an effective cutoff frequency of $6 \mathrm{~Hz}$, as suggested by Winter [18].

We found ankle-foot ROS by transforming the COP of the ground GRF into a shank-based coordinate system. Previous studies measuring nondisabled persons' ROS used only sagittal plane marker data to construct the shank-based coordinate system. These previous calculations of ROS also transformed only the sagittal plane components of the COP of the GRF into this planar shank-based coordinate system [6]. The use of only sagittal plane data when studying nondisabled subjects seems justified since the majority of the movement is in this plane. However, in gait of disabled persons, movements in other planes are often more pronounced. For this reason, a 3-D technique was used to describe the shankbased coordinate system and the 3-D coordinates of the COP were transformed into this system.

We created the shank coordinate system for this study using the lateral ankle marker, a virtual marker at the ankle center, and a virtual marker at the knee center. The virtual markers at the ankle and knee centers were calculated with Orthotrak software (Motion Analysis Corporation) based on a static data collection file that also included markers on the medial malleoli (or medial AFO ankle joint) and medial epicondyles of the knee. The shank-based coordinate system was created as follows: a unit vector (v1) was constructed between the ankle center and the lateral ankle marker. A second unit vector (v2) was constructed between the ankle center and the knee center. When calculating ROS for the left side, we created a third unit vector (v3) by normalizing the cross product of v1 and v2. For the right side, we created the v3 by normalizing the cross product of v2 and v1. Finally, we created a fourth unit vector (v4) by normalizing the cross product of v2 and v3. The shank-based coordinate system was created with $\mathrm{v} 3$ as the $x$-axis, $\mathrm{v} 4$ as the $y$-axis, and $\mathrm{v} 2$ as the $z$-axis. A homogeneous transformation matrix was created with the virtual ankle center marker as the origin for the shank-based coordinate system. For each frame of data, the shank-based coordinate system was created and the COP location was transformed from the laboratorybased coordinate system into the shank-based coordinate system. The ankle-foot ROS for this study was defined as the projection of the COP onto the shank-based $x$ - $z$ plane, measured between initial contact (specifically, the first reliable COP measurement immediately following initial contact) and opposite initial contact. Each measured ROS was fitted with the equation for the lower arc of a circle as described previously [6]. Specific parameters examined in this study included the radius and arc length of the best-fit circular arc. Finally, the $x$-value (in shank-based coordinates) of the first reliable COP point was analyzed as a measure of the anterior location of this point with respect to the ankle center. Henceforth, this point is referred to simply as "the first COP point."

Given the very small sample size, assessing with any confidence that the target population was normally distributed was difficult. Statistical analysis indicated that some variables lacked homogeneity of variance (tested with the Levene statistic: ankle angle at midswing and 
arc radius, $p=0.015$ and $p=0.023$, respectively) and were not normally distributed (tested with the ShapiroWilks test: No AFO ankle angle at IC and AFO ROS arc radius, $p=0.044$ and $p=0.005$, respectively). Hence, we approached statistical analyses conservatively and used nonparametric tests. Medians for temporospatial, kinematic, and ROS data are presented, along with first and third quartiles. We used Mann-Whitney tests to compare independent groups (e.g., subjects with hemiplegia with and without AFO vs control subjects) with Bonferroni-adjusted significance level set at $\alpha \leq 0.025$. We used Wilcoxon signed-rank tests to compare dependent groups (No AFO vs AFO) with significance set at $\alpha \leq 0.05$.

\section{RESULTS}

The 13 subjects with hemiplegia ranged in age from 43 to 66 years (mean \pm standard deviation $51.5 \pm$ 6.8 years) with a mean time since stroke of $8.2 \pm 4.5$ years. Six were female and seven were male; three had right hemiplegia and ten had left hemiplegia. Three additional hemiplegic subjects were excluded because their
ROS could not be fit accurately with the lower arc of a circle. ROS for these subjects was more closely represented by the upper arc of a circle (i.e., they were concave down), causing our fitting routine to indicate negative radii. Control subjects had a mean age of $57.1 \pm 8.5$ years, with four females and eight males. Table 1 is a summary of data on the subjects with hemiplegia, including clinical examination results. Data are absent for one subject who declined to participate in this part of the evaluation. Clinical examination results suggested that all subjects with hemiplegia had some gastrocnemius tightness, because all were unable reach a neutral ankle position on passive manipulation of the involved ankle with the knee extended. However, gait data (specifically maximum dorsiflexion during stance) indicated that only one subject lacked dorsiflexion ROM. Table 2 summarizes the temporospatial, kinematic, and ROS results.

For normal self-selected walking speed and regardless of AFO use, control subjects walked significantly faster than the hemiplegic subjects $(p<0.001)$ (Figure 2(a)). However, compared to the hemiplegic subjects normal speed, no significant difference was noted in walking speed when control subjects walked at a very slow self-selected walking speed $(p=0.852$ without

Table 1.

Summary of subject data (mean \pm 1 standard deviation [SD]).

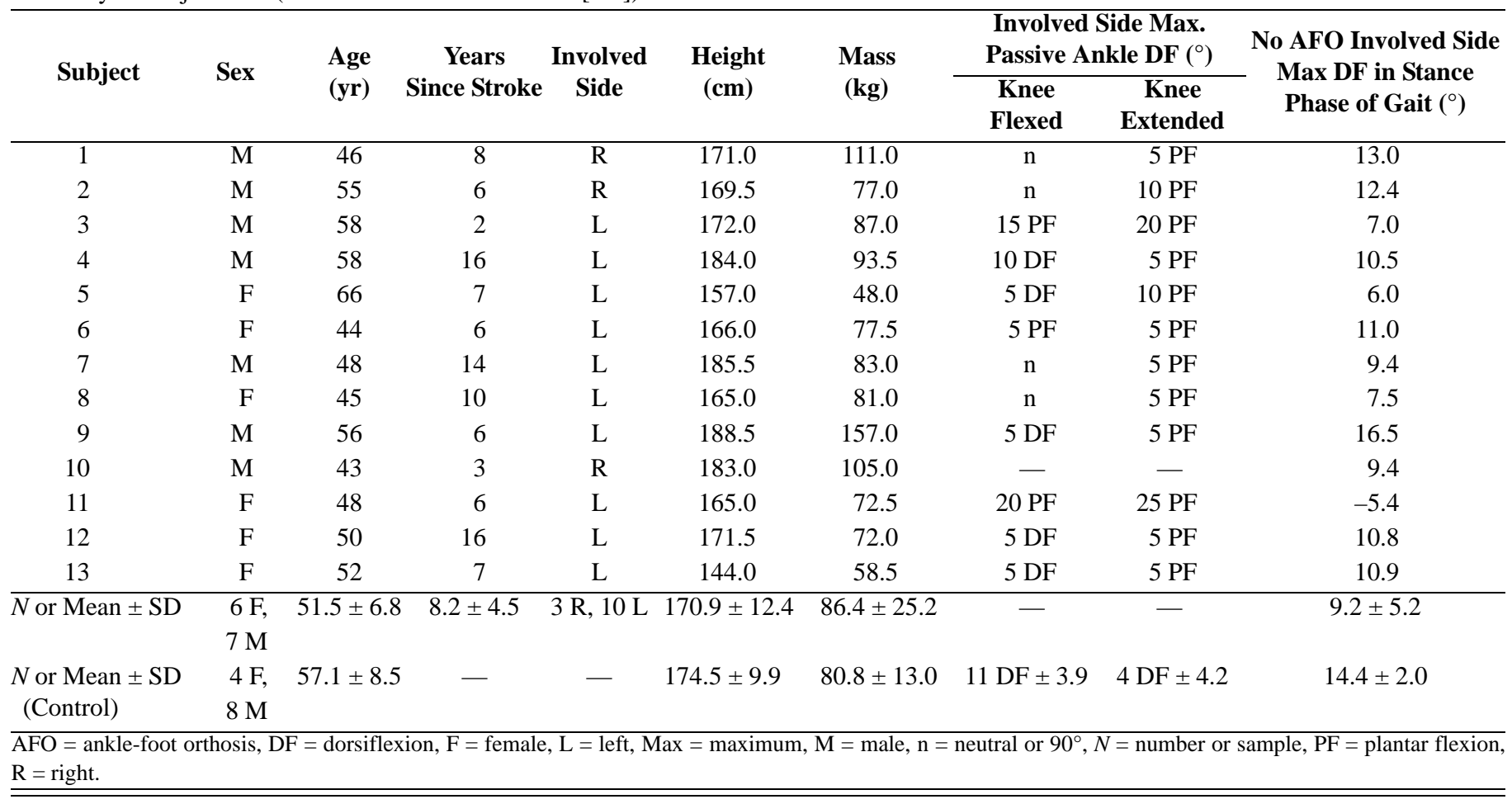


Table 2.

Summary of temporospatial, kinematic, and roll-over shape (ROS) results for control group and subjects with hemiplegia: Data shown as median (1st quartile, 3rd quartile).

\begin{tabular}{|c|c|c|c|c|c|c|}
\hline \multirow{2}{*}{ Variable } & \multicolumn{2}{|c|}{ Control } & \multicolumn{4}{|c|}{ Hemiplegic (Normal Speed) } \\
\hline & Normal & Very Slow & \multicolumn{2}{|c|}{ No AFO } & \multicolumn{2}{|c|}{ AFO } \\
\hline Speed (m/s) & $1.18(1.08,1.35)$ & $0.63(0.57,0.65)$ & \multicolumn{2}{|c|}{$0.57(0.48,0.72)$} & \multicolumn{2}{|c|}{$0.63(0.46,0.72)$} \\
\hline Step Width $(\mathrm{cm})$ & - & $13.8(10.7,14.9)$ & \multicolumn{2}{|c|}{$18.8(17.9,22.8)$} & \multicolumn{2}{|c|}{$17.6(15.6,19.6)$} \\
\hline \multirow{2}{*}{$\begin{array}{l}\text { Step Length Symmetry Index } \\
\text { (SL minus IL) }\end{array}$} & - & - & \multicolumn{2}{|c|}{$-6.7(-10.3,-3.9)$} & \multicolumn{2}{|c|}{$-0.9(-5.3,3.2)$} \\
\hline & & & $\mathrm{IL}$ & SL & IL & SL \\
\hline Step Length (cm) & - & $53.0(47.9,54.3)$ & $44.0(37.0,62.0)$ & $40.5(36.2,51.7)$ & $44.7(35.4,58.6)$ & $43.8(37.7,54.5)$ \\
\hline Ankle Angle at IC $\left({ }^{\circ}\right)^{*}$ & - & $-1.1(-1.6,-0.2)$ & $-12.1(-15.0,-8.9)$ & - & $1.4(-0.1,2.8)$ & - \\
\hline Ankle Angle at Midswing $\left({ }^{\circ}\right)^{*}$ & - & $4.3(3.0,6.0)$ & $-7.6(-10.1,-0.2)$ & - & $2.2(-0.3,5.7)$ & - \\
\hline ROS Arc Radius (\% foot length) & - & $100.9(90.0,105.8)$ & $67.4(49.2,82.4)$ & - & $139.3(95.3,278.6)$ & 一 \\
\hline ROS Arc Length (\% foot length) & - & $64.6(62.5,69.3)$ & $32.6(25.8,45.3)$ & - & $55.7(48.3,66.4)$ & - \\
\hline $\begin{array}{l}\text { Sagittal Location of 1st COP } \\
\text { Point (\% foot length) }{ }^{\dagger}\end{array}$ & - & $-16.0(-16.6,-13.7)$ & $-1.2(-6.4,10.8)$ & - & $-20.0(-24.3,-18.4)$ & - \\
\hline
\end{tabular}

AFO and $p=0.538$ with AFO). Since kinematic and kinetic data are affected by speed, a speed-matched comparison (i.e., normal walking speed for the hemiplegic subjects and very slow walking speed for the control subjects) was used for all subsequent analyses. The normal self-selected walking speed of the hemiplegic subjects walking with and without an AFO was not significantly different ( $p=0.507)$.

At the ankle, the AFO (compared with No AFO) significantly decreased the plantar flexion angle to neutral at initial contact ( $p=0.001)$ and significantly altered the angle at midswing from plantar flexion to slight dorsiflexion ( $p=0.012)$ (Figure 2(b) and (c)). Compared with control subjects, the ankle of subjects with hemiplegia was significantly more plantar flexed at initial contact and midswing without an AFO ( $p=0.001$ and $p=0.000$, respectively). With an AFO, the ankle was significantly more dorsiflexed at initial contact compared with control subjects ( $p=0.005)$, but no significant difference was found in ankle angle at midswing ( $p=0.137)$.

Without an AFO, median step length was shorter on the sound side compared with the involved side. This asymmetry decreased significantly when an AFO was worn $(p=0.007)$ because of a significant increase in sound limb step length ( $p=0.033$ for the sound limb and $p=0.116$ for the involved limb) (Figure 3). Step width was significantly reduced $(p=0.016)$ when subjects with hemiplegia used the AFO, although it remained significantly greater than that of control subjects $(p=0.007)$ (Figure 4).
Bilateral, median ROS for all subjects and each condition are shown in Figure 5. For the involved limb, use of the AFO (compared with No AFO) significantly increased the ROS arc radius and length ( $p=0.001$ and $p=0.002$, respectively) and significantly altered the sagittal plane location of the first COP point, moving it posterior to the ankle joint center ( $p=0.001$ ) (Figure 6(a) and (b)). Arc length and arc radius were no different to those of control subjects when subjects with hemiplegia walked with an AFO ( $p=0.152$ and $p=0.035$, respectively). The AFO condition resulted in the first COP point moving posterior to the ankle joint compared with a more anterior position without the AFO. Additionally, the first COP point was located significantly more posterior than that of the control subjects $(p=0.022)$ (Figure 6(c)). When walking without an AFO, progression of the COP changed direction twice during midstance. This change of direction was eliminated when the AFO was worn. The AFO resulted in more uniform forward progression of the COP compared with subjects walking without an AFO.

\section{DISCUSSION}

The AFO tested in this study improved ROS, resulting in an arc length and radius that were no different from those of control subjects. However, the location of the first COP point was significantly more posterior compared with that of control subjects. When subjects walked without an AFO (shoes only), initial contact by the subjects with 
(a)

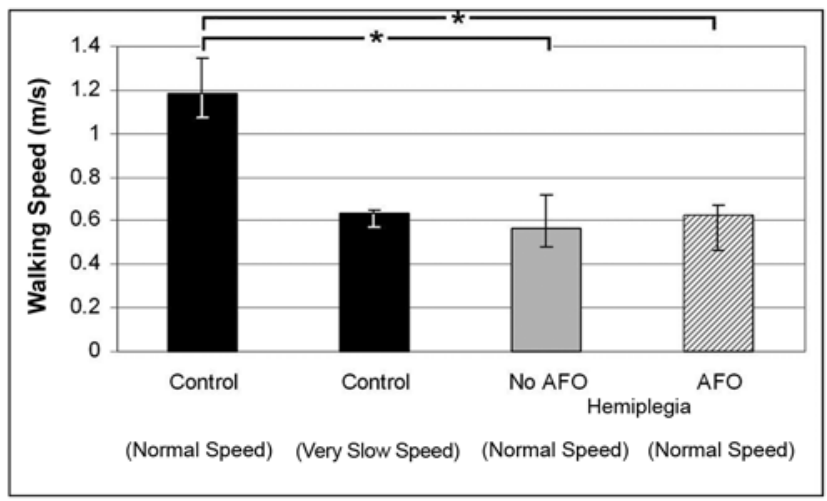

(b)

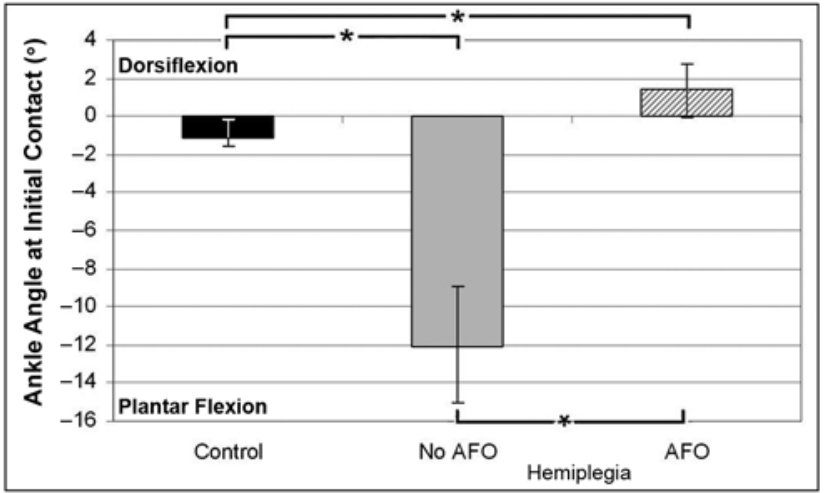

(c)

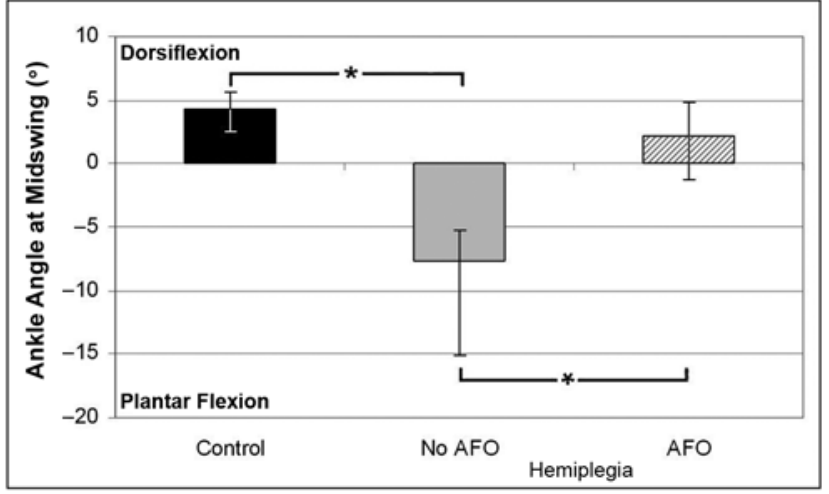

Figure 2.

(a) Median walking speed for control group and subjects with hemiplegia with and without ankle-foot orthosis (AFO) (normal speed: $p_{\text {Control:NoAFO }}<0.001, p_{\text {Control:AFO }}<0.001, p_{\text {NoAFO:AFO }}=$ 0.507 ; very slow speed: $\left.p_{\text {Control: } \mathrm{NoAFO}}=0.852, p_{\text {Control } \mathrm{AFO}}=0.538\right)$. Median ankle angle at (b) initial contact and (c) midswing for control subjects and involved limb of subjects with hemiplegia with and without AFO (initial contact: $p_{\text {Control:NoAFO }}=0.001, p_{\text {Control: } \mathrm{AFO}}=$ $0.005, p_{\mathrm{NoAFO}: \mathrm{AFO}}=0.001$; midswing: $p_{\text {Control:NoAFO }}<0.001$, $\left.p_{\text {Control: } \mathrm{AFO}}=0.137, p_{\mathrm{NoAFO}} \mathrm{AFO}=0.001\right)$. Stars indicate statistically significant difference. Variance indicated by 1 st and 3rd quartiles.

hemiplegia was with the entire foot at once, or forefoot first and then the heel, rather than the "heel-toe gait" exhibited by nondisabled ambulators. In this situation, the arc length (a)

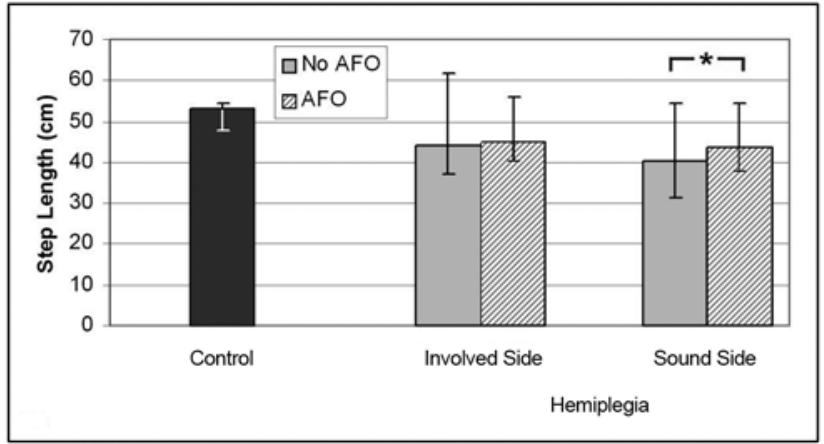

(b)

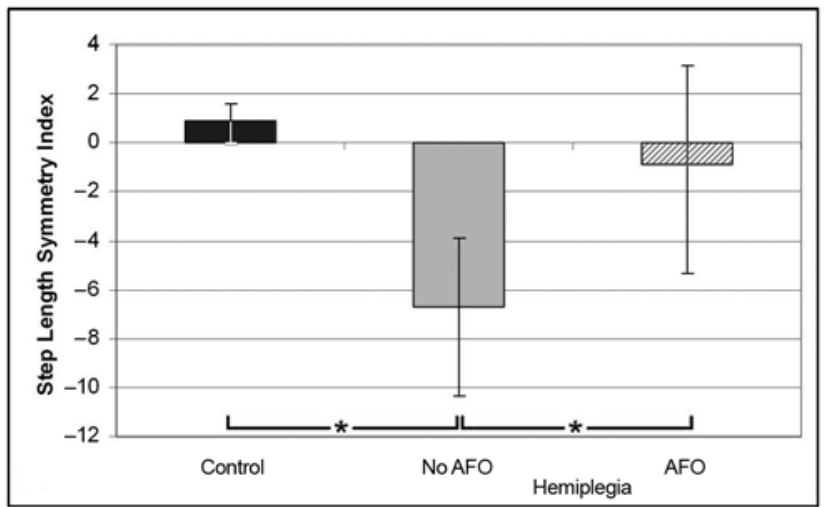

Figure 3.

(a) Median step length and (b) step length symmetry for control group and subjects with hemiplegia with and without ankle-foot orthosis (AFO) (step length: $p_{\mathrm{NoAFO}: \mathrm{AFO}}=0.033$; step length symmetry: $\left.p_{\text {Control: } \mathrm{NoAFO}}=0.016, p_{\text {Control: } \mathrm{AFO}}=0.611, p_{\mathrm{NoAFO}: \mathrm{AFO}}=0.007\right)$. For control group, step length was mean of right and left steps for each individual subject. For subjects with hemiplegia, step-length symmetry was calculated as sound limb minus involved limb and for control subjects as right limb minus left limb. Stars indicate statistically significant difference. Variance indicated by 1st and 3rd quartiles.

of the ROS was shortened and the COP point moved back and forth beneath the foot during the first half of stance rather than progressing anteriorly in an uninterrupted manner. When subjects with hemiplegia used the AFO, a perturbation in the COP progression during midstance was still present, although less pronounced, which implies that forward progression of the body over the foot continued to be disrupted despite significant increases in ROS arc length and radius.

Although the AFO significantly altered the ankle angle in stance and swing so that it was closer to that of control subjects, it did so by mechanically blocking plantar flexion motion. The disruption in forward progression suggested by motion of the COP may be due to spasticity, inextensibility, and inappropriate activation of the plantar 


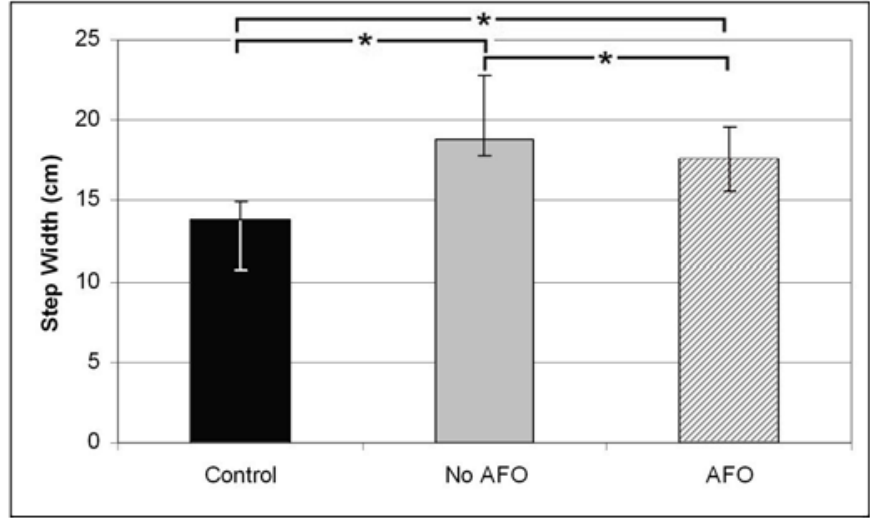

Figure 4.

Median step width for control group and subjects with hemiplegia walking with and without ankle-foot orthosis (AFO) ( $p_{\text {Control:NoAFO }}$ $\left.<0.001, p_{\text {Control:AFO }}=0.007, p_{\text {NoAFO:AFO }}=0.016\right)$. Stars indicate statistically significant difference. Variance indicated by 1 st and 3rd quartiles.

flexors. Clinical examination indicated that all the subjects had some gastrocnemius tightness, but gait data suggested that only one subject lacked dorsiflexion range. However, a number of issues must be considered when comparing these results. The results of passive ROM testing depend on the physical strength of the tester, and the force applied by the tester is likely to be less than that applied by body weight when the subject is walking. The length of time the stretch is applied should also be considered; for example, Hesse et al. used a 10-minute standing test to rule out plantar flexion contracture $[4,19]$. In our study, the stretch applied during the passive ROM test was considerably shorter, and apparently the passive ROM test underestimated the actual ankle ROM available to each subject.

Passive ankle ROM measured during the clinical exam and sagittal ankle motion measured by the motion analysis system during walking may not be measuring the same motion. We took care during the clinical exam to ensure that dorsiflexion motion of the anatomical ankle joint was isolated from midfoot motion. However, when subjects with hemiplegia walked without an AFO, markers were placed both on the shoe (toe and calcaneal markers) and on the malleoli. Hence, ankle motion during gait is a combination of anatomical joint motion and any motion between the foot and the shoe. Motion of the midfoot could also have affected the measured dorsiflexion. When subjects walked with an AFO, markers used to measure ankle motion were placed on the anatomical knee joint, orthotic ankle joint, and shoe. In this case, ankle motion
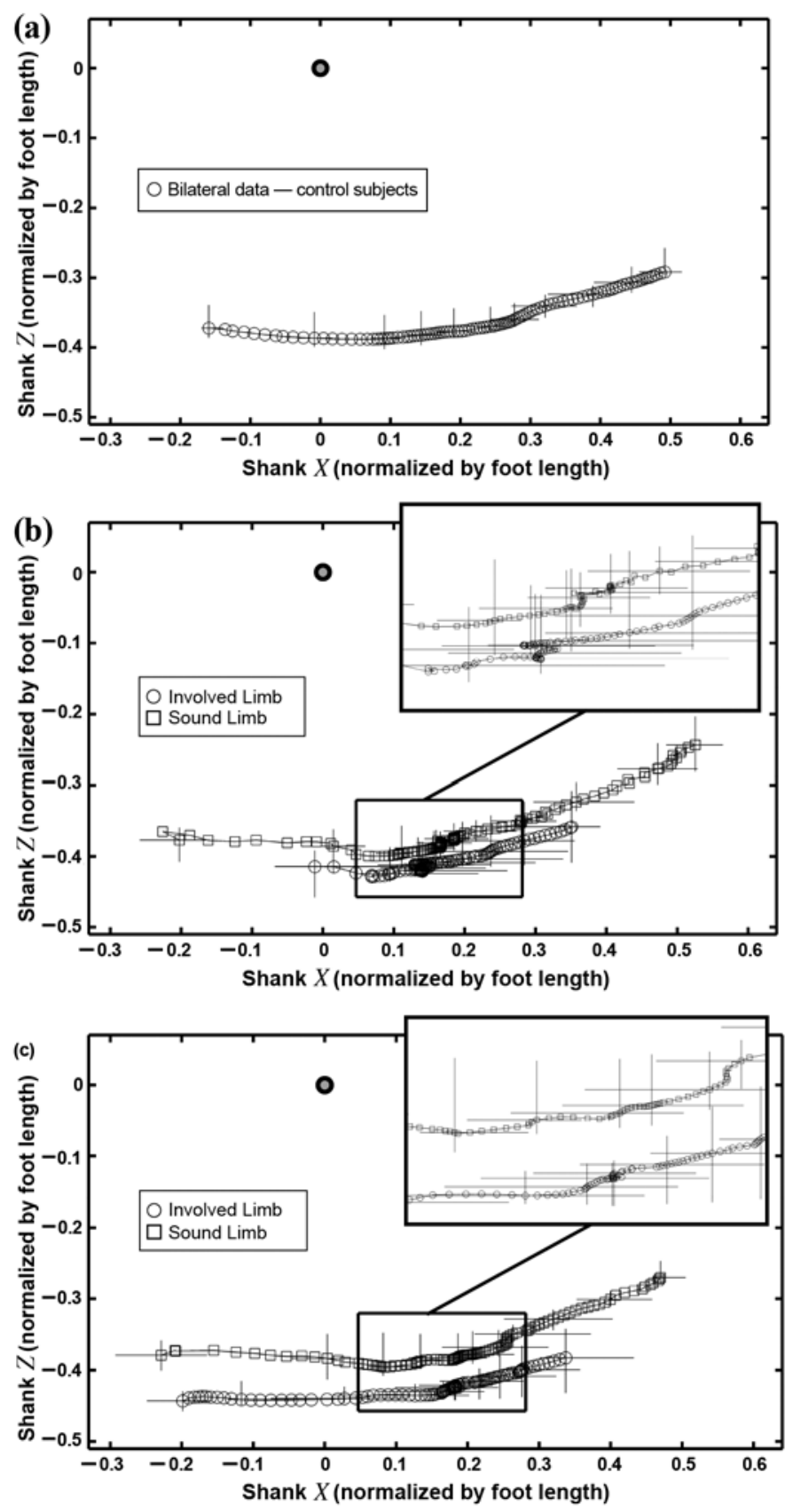

Figure 5.

Median roll-over shapes for (a) control and (b) groups with hemiplegia walking without and (c) with the ankle-foot orthosis. Inset pictures highlight center of pressure motion in midstance. Variance indicated by 1 st and 3rd quartiles.

during gait is a combination of anatomical motion, motion of the orthosis, and motion between the shoe and AFO.

When subjects used the AFO, the location of the first COP point moved further posterior to the ankle joint center. This result was likely caused by the AFO as it facilitated 
(a)

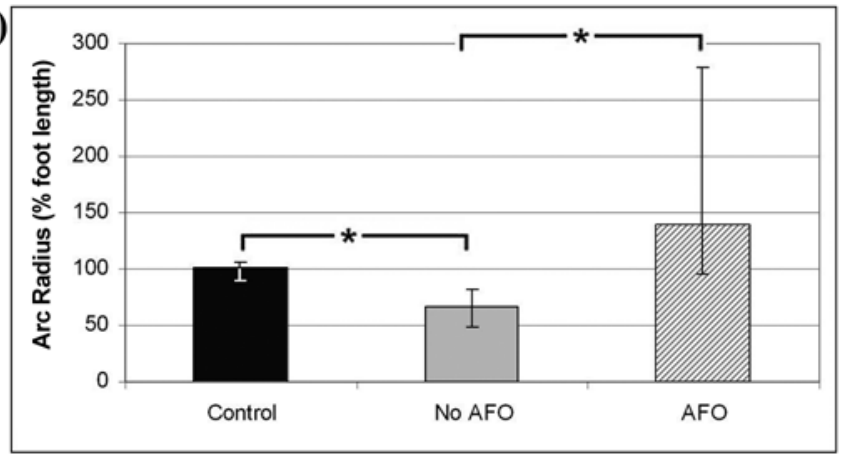

(b)

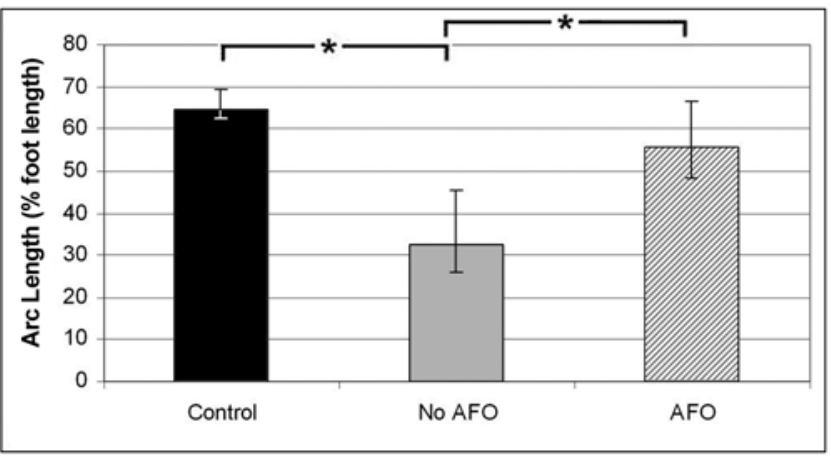

(c)

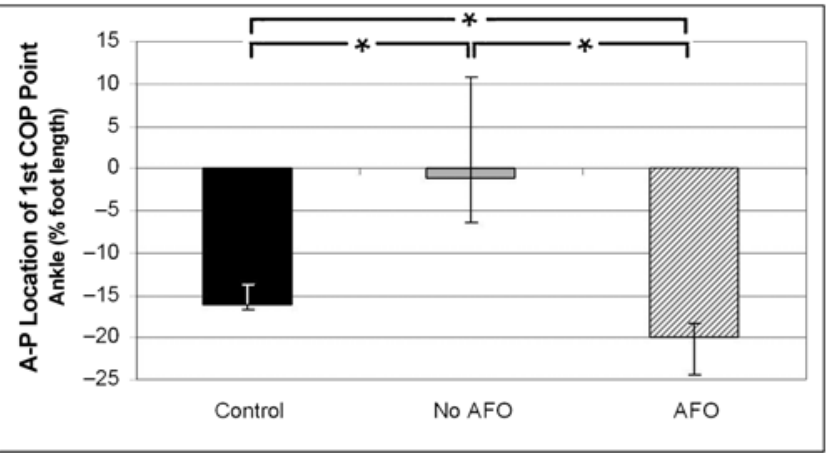

Figure 6.

(a) Median roll-over shape (ROS) arc radius, (b) ROS arc length, and (c) sagittal plane (anterior-posterior [A-P]) location of first center of pressure (COP) point with respect to ankle for control group and subjects with hemiplegia involved limb walking with and without ankle-foot orthosis (AFO) (arc radius: $p_{\text {Control:NoAFO }}=0.002$, $p_{\text {Control: } \mathrm{AFO}}=0.035, p_{\mathrm{NoAFO}: \mathrm{AFO}}=0.001$; arc length: $p_{\text {Control: } \mathrm{NoAFO}}=$ $0.001, p_{\text {Control:AFO }}=0.152 ; p_{\text {NoAFO:AFO }}=0.002$; COP location: $p_{\text {Control:NoAFO }}<0.001, p_{\text {Control:AFO }}=0.022, p_{\text {NoAFO:AFO }}=0.001$ ). Data were normalized by foot length. Stars indicate statistically significant difference. Variance indicated by 1st and 3rd quartiles.

initial contact with the heel. However, the AFO may have overcorrected the problem. This overcorrection may be due in part to the orthosis severely limiting the plantar flexion that occurs normally during first rocker, causing the subject to initiate contact more posteriorly and to pivot on the heel until the tibia has moved far enough anteriorly to allow the rest of the foot to contact the ground. Additional compliance might be achieved by use of a plantar flexion bumper or a "cushion heel." Either of these components may help move the first point of the COP more anteriorly by allowing some plantar flexion (actual or simulated) in early stance. However, this modification to the design of the AFO needs to be achieved in a way that does not compromise control of the knee, especially where hyperextension may be present. We are not aware of any AFO component or orthotic device currently available that allows plantar flexion during loading response but resists it during midstance (limiting hyperextension of the knee).

Walking is a process of getting from one point to another as safely and efficiently as possible and fast enough for one to function in society. Despite significant increases in ROS arc length and radius with an AFO, we did not see any changes in the subjects' self-selected walking speed. Having a ROS that was closer to normal did not impact their ability to get from one point to another any more quickly. Many factors impact walking speed, including the ability to advance the body over the stance limb, to weight shift quickly and safely from one limb to the other, and to flex the knee at the appropriate time and with adequate velocity and magnitude. Stroke affects all these factors, and they were not necessarily addressed by the AFO design used in this study. These factors may not be influenced by ROS, although the smoothing of the COP progression when the AFO was worn would imply some improvement in forward progression. Improving walking speed may be possible with the use of an AFO designed to improve terminal stance kinematics, especially hip and knee extension. Research has suggested that nonarticulated AFOs with appropriate alignment or an AFO with a dorsiflexion stop might improve walking speed [20]. Investigation of the ROS of other AFO designs is warranted to further explore this issue. Additionally, improvements in ROS resulting from AFO use may have a greater impact in conditions that solely affect the ankle and foot as opposed to a complex pathology such as hemiplegia that affects multiple levels.

The robotics literature suggests that an appropriate ROS may improve stability: Wisse and van Frankenhuyzen showed that a mechanical model with a zero radius rocker could not tolerate disturbances without falling down while mechanical models with rockers could tolerate increasingly larger disturbances as the radius of the rocker increased [21]. If the same relationship is true in humans, 
then perhaps an appropriate ROS would provide some level of inherent stability during walking.

Step width is often considered clinically as an indicator of stability since step width is associated with the size of the base of support in the coronal plane. In this study, subjects with hemiplegia ambulated with a significantly greater step width than control subjects. Increasing the base of support improves stability by providing a larger zone with respect to which the body center of mass may be located and allow the person to remain upright. Mechanical work is performed to redirect the center of mass during the transition between steps [22]. Donelan et al. reported that the preferred step width in nondisabled humans is 13 percent of leg length and that the mechanical and metabolic costs of walking increase with steps that are both wider and narrower than the preferred step width [23]. Donelan et al. also studied the mechanical and metabolic requirements for active lateral stabilization in human walking by providing external lateral stabilization to walking subjects and reported that external stabilization reduced foot placement variability for both preferred and prescribed step width conditions [24]. They suggested that lateral instability affects the choice of preferred step width. Gabell and Nayak suggested that an increase in mean step width indicates a lack of compensation for disturbances causing instability [25]. Heitmann et al. demonstrated a negative correlation between balance performance and step width means and variability in elderly females [26]. If step width indicates stability, then the significant decrease in step width that occurred when the AFO was worn might suggest improved stability. Further research is required to explore this hypothesis.

ROS provides us with a method to quantify the ankle-foot rockers described by Perry [5] and explore the contribution of ankle-foot function to walking. However, at present some limitations exist to applying the ROS analyses to data from subjects with hemiplegia: our ability to compute meaningful radii and arc lengths is compromised for flat shapes and concave-down shapes (leading to the exclusion of three subjects from this study). The circular arc-fitting routine is meant for the lower arc of a circle and works well for concave-up shapes as found in nondisabled subjects. Flat shapes have an infinite radius and cannot be solved. As currently defined, ROS describes function of the ankle-foot complex only while it is "rolling over" and not when it is being unloaded during the double support phase of terminal stance. Giuliani suggested that the greatest loss of motor control of the hemiplegic limb occurs at phase transitions, such as during the transfer of weight from one limb to the other [1]. For this reason, further investigation of the COP during unloading of the affected limb may be warranted.

\section{CONCLUSIONS}

Use of the AFO design tested in this study significantly improved subjects' ROS compared with walking without an AFO, in particular increasing arc radius and length, but did not completely normalize it for the subjects tested, since the location of the first COP point with respect to the ankle center was further posterior than normal. At present, some limitations exist to modeling the ROS of subjects with hemiplegia as the lower arc of a circle since some of the data from hemiplegic subjects cannot be adequately represented by this model (e.g., some of the ROS are concave-down or are flat). Additional investigation is required to see if we can further improve ROS by altering AFO design and allow users to further increase step length and walking speed.

\section{ACKNOWLEDGMENTS}

Data for this project were collected at the VACMARL of the Jesse Brown VA Medical Center, Chicago, Illinois. We would like to acknowledge Dudley Childress, PhD; Steven Gard, PhD; Michael Brncick, MEd, CPO; and Bryan Malas, MHPE, CO, who all contributed invaluable advice during the writing of this article.

This material was based on work supported by the VA Office of Research and Development (R\&D), Rehabilitation R\&D Service, merit review grant A2676I.

The authors have declared that no competing interests exist.

\section{REFERENCES}

1. Giuliani CA. Adult hemiplegic gait. In: Smidt GL, editor. Gait in rehabilitation: Clinical physical therapy. New York (NY): Churchill Livingstone Inc; 1990. p. 253-66.

2. Da Cunha IT Jr, Lim PA, Qureshy H, Henson H, Monga T, Protas EJ. Gait outcomes after acute stroke rehabilitation with supported treadmill ambulation training: A randomized controlled pilot study. Arch Phys Med Rehabil. 2002; 83(9):1258-65. [PMID: 12235606$]$ 
3. Perry J. The mechanics of walking in hemiplegia. Clin Orthop Relat Res. 1969;63:23-31. [PMID: 5769371]

4. Hesse S, Werner C, Matthias K, Stephen K, Berteanu M. Non-velocity-related effects of a rigid double-stopped ankle-foot orthosis on gait and lower limb muscle activity of hemiparetic subjects with an equinovarus deformity. Stroke. 1999;30(9):1855-61. [PMID: 10471436]

5. Perry J. Gait analysis: Normal and pathological function. Thorofare (NJ): SLACK; 1992.

6. Hansen AH, Childress DS, Knox EH. Roll-over shapes of human locomotor systems: Effects of walking speed. Clin Biomech (Bristol, Avon). 2004;19(4):407-14. [PMID: 15109762]

7. Hansen AH, Childress DS. Effects of adding weight to the torso on roll-over characteristics of walking. J Rehabil Res Dev. 2005;42(3):381-90. [PMID: 16187250]

8. Hansen AH, Childress DS. Effects of shoe heel height on biologic rollover characteristics during walking. J Rehabil Res Dev. 2004;41(4):547-54. [PMID: 15558383]

9. Weiss WB, Mulroy SJ, Gronley JK, Perry J, Boyd L. Rigid AFO impairs walking ability in individuals with hemiparesis from CVA [abstract]. Gait Posture. 2002;16:S2.

10. Fatone S, Hansen AH, Gard SA, Malas BS. Effects on gait of ankle alignment and foot-plate length in ankle-foot orthoses (AFOs). Proceedings of the American Academy of Orthotists and Prosthetists annual meeting and scientific symposium; 2005 Mar 16-19; Orlando, FL. Alexandria (VA): American Academy of Orthotists and Prosthetists; 2005.

11. Cook T, Cozzens B. The effects of heel height and anklefoot-orthosis configuration on weight line location: A demonstration of principles. Orthot Prosthet. 1976;30:43-46.

12. Lee KH, Johnston R. Effect of below-knee bracing on knee movement: Biomechanical analysis. Arch Phys Med Rehabil. 1974;55(4):179-82. [PMID: 4823070]

13. Lehmann JF, Ko MJ, deLateur BJ. Knee moments: Origin in normal ambulation and their modification by doublestopped ankle-foot orthoses. Arch Phys Med Rehabil. 1982;63(8):345-51. [PMID: 7436716]

14. Butler PB, Nene AV. The biomechanics of fixed ankle-foot orthoses and their potential in the management of cerebral palsied children. Physiotherapy. 1991;77(2):81-88.

15. Owen E, Bowers R, Meadows B. Tuning of AFO-footwear combinations for neurological disorders. In: Boone D, editor. Proceedings of the 11th World Congress of the International Society for Prosthetics and Orthotics; 2004 Aug 1-6; Hong Kong, China. Hong Kong (China): Hong Kong National Society of the International Society for Prosthetics and Orthotics; 2004.
16. Owen E. Shank angle to floor measures of tuned 'anklefoot orthosis footwear combinations' used with children with cerebral palsy, spina bifida and other conditions. Gait Posture. 2002;16:S132-33.

17. Gard SA, Fatone S. Biomechanics of lower limb function and gait. In: Condie E, Campbell J, Martina J, editors. Report of a consensus conference on the orthotic management of stroke patients; 2005 Sep 21-26, Ellecom, the Netherlands. Copenhagen (Denmark): International Society for Prosthetics and Orthotics; 2004.

18. Winter DA. Biomechanics and motor control of human movement. New York (NY): Wiley; 1990.

19. Hesse S, Luecke D, Jahnke MT, Mauritz KH. Gait function in spastic hemiparetic patients walking barefoot, with firm shoes, and with ankle-foot orthosis. Int J Rehabil Res. 1996; 19(2):133-41. [PMID: 8842827]

20. Bowers RJ. Non-articulated ankle-foot orthoses. In: Condie E, Campbell J, Martina J, editors. Report of a consensus conference on the orthotic management of stroke patients. 2005 Sep 21-26; Ellecom, the Netherlands. Copenhagen (Denmark): International Society for Prosthetics and Orthotics; 2004.

21. Wisse M, Van Frankenhuyzen J. Design and construction of MIKE: A 2D autonomous biped based on passive dynamic walking. Proceedings of the 2nd International Symposium on Adaptive Motion of Animals and Machines; 2003 Mar 4-5; Kyoto, Japan. Available from: http://www.kimura.is.uec.ac.jp/ amam2003/online-proceedings.html

22. Kuo AD, Donelan JM, Ruina A. Energetic consequences of walking like an inverted pendulum: Step-to-step transitions. Exerc Sport Sci Rev. 2005;33(2):88-97. [PMID: 15821430]

23. Donelan J, Kram R, Kuo A. Mechanical and metabolic determinants of the preferred step width in human walking. Proc Royal Soc London Biol Sci. 2001;268(1480):1985-92.

24. Donelan JM, Shipman DW, Kram R, Kuo AD. Mechanical and metabolic requirements for active lateral stabilization in human walking. J Biomech. 2004;37(6):827-35. [PMID: 15111070]

25. Gabell A, Nayak US. The effect of age on variability in gait. J Gerontol. 1984;39(6):662-66. [PMID: 6491179]

26. Heitmann DK, Gossman MR, Shaddeau SA, Jackson JR. Balance performance and step width in noninstitutionalized, elderly, female fallers and nonfallers. Phys Ther. 1989; 69(11):923-31. [PMID: 2813520]

Submitted for publication August 11, 2006. Accepted in revised form November 29, 2006. 